\title{
Effect of predominant breastfeeding duration on infant growth: a prospective study using nonlinear mixed effect models
}

\author{
Maria Helena Constantino Spyrides, ${ }^{1}$ Cláudio José Struchiner, ${ }^{2}$ \\ Maria Tereza Serrano Barbosa, ${ }^{3}$ Gilberto $\mathrm{Kac}^{4}$
}

\begin{abstract}
Objective: The aim of this study is to assess the effect of predominant breastfeeding duration on infant growth by means of repeated measurements model.

Methods: This prospective study is comprised of four follow-up evaluations at approximately $0.5,2,6$ and 9 months after birth, including structured interviews that simultaneously gathered information regarding infant growth and breastfeeding practices. The study took place in a healthcare center in Rio de Janeiro, Brazil, from 1999 to 2001. Four hundred seventy-nine postpartum women and their newborns were enrolled in the cohort. Body weight and length measurements taken at five different occasions (birth, 0.5, 2, 6, and 9 months) constituted the dependent variables. We expressed the growth process using nonlinear mixed models.

Results: Infants with longer predominant breastfeeding duration, although growing faster in the first months of life, reached an inferior equilibrium body weight and length compared to infants who received nonhuman milk earlier in life. The age at which the rate of weight gain of the formula-fed infants becomes greater than that of the breastfed infants is approximately 6.75 months for boys and 7 months for girls.

Conclusions: This study confirms the differences observed in infant growth according to different breastfeeding practices starting from the sixth month of life. Use of nonlinear models allowed for a greater precision of parameter estimates. We believe that this approach facilitates the analysis and interpretation of growth data at the individual and population levels.
\end{abstract}

J Pediatr (Rio J). 2008;84(3):237-243: Breastfeeding, infant growth, nonlinear mixed models, repeated measures.

\section{Introduction}

Infant growth patterns vary substantially as a function of several factors comprising determinants such as nutritional, cultural, environmental and social conditions, as well as biological and genetic factors. In the first 6 months of life, the most important source of nutrients is breast milk. As a consequence, it is important to know how breastfeeding duration influences the increase in body weight and length in infants.

The effect of breastfeeding on infant growth has been studied by several authors. ${ }^{1-6}$ Several researchers have verified that breastfed infants show a higher growth rate early in

1. Doutora. Professora adjunta, Departamento de Estatística, Universidade Federal do Rio Grande do Norte (UFRN), Natal, RN, Brazil.

2. Doutor. Professor adjunto, Universidade do Estado do Rio de Janeiro (UERJ), Rio de Janeiro, RJ, Brazil. Pesquisador titular, Escola Nacional de Saúde Pública, Fundação Oswaldo Cruz (FIOCRUZ), Rio de Janeiro, RJ, Brazil.

3. Doutora. Professora adjunta, Universidade Federal do Estado do Rio de Janeiro (UNIRIO), Rio de Janeiro, RJ, Brazil.

4. Doutor. Professor, Instituto de Nutrição Josué de Castro, Departamento de Nutrição Social Aplicada, Universidade Federal do Rio de Janeiro (UFRJ), Rio de Janeiro, RJ, Brazil.

Finnantial support: Fundação Universitário José Bonifácio (FUJB), Rio de Janeiro, RJ, Brazil, and Fundação de Amparo à Pesquisa do Estado do Rio de Janeiro (FAPERJ), Rio de Janeiro, RJ, Brazil.

No conflicts of interest declared concerning the publication of this article.

Suggested citation: Spyrides MH, Struchiner CJ, Barbosa MT, Kac G. Effect of predominant breastfeeding duration on infant growth: a prospective study using nonlinear mixed effect models. J Pediatr (Rio J). 2008;84(3):237-243.

Manuscript received Dec 3 2007, accepted for publication Mar 102008.

doi:10.2223/JPED.1797 
life when compared to formula-fed infants. Other studies ${ }^{1,6,7}$ have reported that the rate of weight gain in formula-fed infants becomes greater than that of breastfed infants at some point during the first few months of growth. There is still an undergoing debate on the issue, especially after the release in 2006 of the World Health Organization (WHO) new standards to asses the growth and development of children under 5 years. $^{8,9}$

The most common anthropometric measurements to assess infant growth are body weight and length. When these measurements are taken repeatedly on the same individual over time, it renders the analysis of the growth process more consistent. It is important to mention that it is difficult or even impossible to quantify the variability attributed to environmental factors in observational studies. As a result, the statistical analysis of these data sets requires models that account for other sources of variation to take into account the uncontrollable factors. In this regard, the use of nonlinear mixed-effects models is a novel approach. ${ }^{10}$

The aim of this study is to assess the effect of predominant breastfeeding duration on infant growth, using data gathered in a health center in Rio de Janeiro, Brazil. The approach of the present study improves on the methodology hitherto used to describe growth patterns among infants, since none of the studies cited previously has analyzed the breastfeeding effect on infant growth using nonlinear mixed models. This approach might facilitate the analysis and interpretation of growth data at both the individual and population levels.

\section{Methods}

\section{Study design}

The data set used in this analysis comes from a research project that evaluated body composition and maternal obesity, simultaneously gathering information about infant growth and breastfeeding practices. Criteria for recruitment and selection are available in other publications. ${ }^{11-15} \mathrm{~A}$ prospective cohort of Brazilian women aged 15 to 45 years living in the city of Rio de Janeiro, Brazil, was followed for 9 months. Data collection lasted 24 months (15 months for recruitment plus 9 months for follow-up), from May 1999 to April 2001. Four hundred seventy-nine women and their newborns were enrolled in the cohort. This prospective study includes four follow-up evaluations at approximately $0.5,2,6$ and 9 months after birth. In addition to these evaluations, weight and length at birth were also observed comprising five repeated measurements over time.

\section{Inclusion and exclusion criteria}

The eligibility criteria for inclusion in the cohort were: a) infants born alive from mothers ranging from 15 to 45 years of age; b) infants being less than 1 month old by the time of the first interview; c) mothers with no chronic illnesses, d) not being the mother of twins and e) living within the catchment area of Marcolino Candau Municipal Health Center in Rio de Janeiro, Brazil.

Infants whose anthropometric indexes lied outside the normal biological range as specified in the guideline of the $\mathrm{WHO}^{16}$ were excluded. None of the children in this study scored outside of these bounds, but those who lacked information about breastfeeding duration or had too little information regarding body weight and length were excluded.

\section{Study variables}

Infants' body weight and length data were obtained in four follow-up evaluation sessions, as mother and child visited the healthcare center during the 9-month follow-up period. Body weight and length at birth were reported by mothers at the moment of the first interview. The anthropometric measures taken at five occasions (birth, 0.5, 2, 6, and 9 months) constituted the dependent variable of the model.

Body weight was measured with the infant positioned on the mother's lap by means of a digital scale (Model PL 150, Filizola S/A, São Paulo, Brazil), accurate to $0.1 \mathrm{~kg}$. The mother was weighed first and then the weight of the infant was obtained by subtraction. Length was measured using the Kiddimetre infantometer (Child Growth Foundation, UK) accurate to $0.1 \mathrm{~cm}$, with the infant lying down, and by following the recommendations of Lohman et al. ${ }^{17}$

Breastfeeding practices were determined following the Pan American Health Organization (OPAS)/WHO ${ }^{18}$ guideline that defines as predominantly breastfed the infants receiving only breast milk and other liquids, such as water, tea or juices. For the analysis, predominant breastfeeding duration (in months) at each follow-up evaluation was treated as the main covariate. Note that this is a time-dependent variable, which means that at each point in time this variable might assume a different value. Otherwise, once the maximum value was reached we kept constant thereafter.

Other covariates studied included age, sex (boys, girls), gestational age (in weeks) and type of delivery (natural or by cesarean delivery). Gestational age was obtained through the information on the date of the last menstrual cycle. Newborns with a gestational age $<37$ weeks at birth were considered as premature. Type of delivery was reported by the mother. Infant age, although measured in days from birth to the corresponding follow-up evaluation, was expressed in months.

\section{Statistical modeling}

Recently, the use of nonlinear mixed effects models has become increasingly common in scientific research. They are particularly useful when dealing with repeated measures with data not equally spaced in time, a data structure which is very common in public health, biology and other areas. Infant 
growth pattern follows a nonlinear trajectory in time. Nonlinear statistical models are, therefore, required to describe this behavior. There are many nonlinear functions that can be used. Based on the observed data, the behavior of these measurements (body weight or length) throughout the analyzed period had the shape of the asymptotic regression function. It means that in the earlier months the growth acceleration is greater, slowing down at the end of the observed period. So, the function that fits best with these prospective data of the infant growth in the first months of life is the asymptotic regression model described by Pinheiro \& Bates. ${ }^{10}$ That model has three parameters and their interpretation is as follows: $P_{\text {asymp. }}$ represents the equilibrium body weight or length, i.e., the equilibrium body weight or length at the end of the study period ( 9 months of age); $P_{0}$ represents the body weight or length at birth, and log_rate is the logarithm of the rate of body weight or length gain.

In this aspect, the nonlinear mixed model becomes an interesting alternative. While in the fixed effects model the same equation applies to each child, demanding therefore the use of a margin of error, in the random effects model parameters vary from child to child, increasing thus the precision of estimated parameters. The variations within and between infants are estimated separately: within-group variation comprises deviations in anthropometric measurements for one infant around his own growth behavior, while between-group variation describes how the coefficients vary from infant to infant.

Several correlation structures were tested, considering two components: one for the random effects and another for the correlation between repeated measurements. It is well known that repeated measurements are autocorrelated, which means that, for example, body weight at time 1 is correlated with body weight at time 2 . For this reason it is necessary to include a correlation parameter. The restricted maximum likelihood estimation method (RMLE) was used to estimate the parameters in the model, using S-PLUS software (MathSoft, Inc, Cambridge, MA). Akaike information criterion (AIC) and likelihood ratio (LR), two procedures to test the model's goodness of fit, were used to help decide which model is best.

The main advantage of the use of nonlinear mixed-effects models is the possibility of employing a nonlinear function to represent the biological mechanism of the process being studied, allowing for parameters that offer a more natural interpretation of the infant's growth. Furthermore, it also allows the estimation of the individual variability from the mean response attributed to the group under study.

The study was approved by the appropriate research ethics committees (Universidade Federal do Rio de Janeiro and Universidade de São Paulo), and signed consent was obtained from each study participant.

\section{Results}

In this study, 479 children were followed. Of these, 63 were measured just once, 88 twice, 90 contributed three measurements, and 238 completed the four interviews. In total, $30 \%$ of the study participants were lost to follow-up. Losses of follow-up have been described as a function of explanatory variables elsewhere, and no selection bias was identified.

The sample of this study included 236 boys and 243 girls, a total of 479 infants in the first follow-up evaluation. Mean birth weight for boys was 3,254.3 $\pm 507.2 \mathrm{~g}$, and for girls, $3,115.0 \pm 478 \mathrm{~g}$. Mean birth length was $49.3 \pm 2.4 \mathrm{~cm}$ and $48.6 \pm 2.4$ for boys and girls, respectively. Mean gestational age was 38.6 weeks, varying from 30 to 42 weeks. The percentages of premature deliveries were 11.3 and $9.0 \%$ for boys and girls, respectively.

At the time of the first interview, $11.5 \%$ of the mothers declared they were predominantly breastfeeding. At 9 months postpartum, $40.0 \%$ of the infants received only formulas and $40.0 \%$ received a mix of breast milk and formulas. Median duration of predominant breastfeeding was 67 days, and $27 \%$ of the mothers' breastfed for 1 month or less. Median exclusive breastfeeding duration was 21 days for premature infants and 90 days for those born in term (results not shown).

Parameter estimates between the groups are similar, despite the fact that the model that considers the random effects presents a smaller standard error. This occurs because the fixed-effects model does not include the variability among groups, which is then absorbed in the residual standard error.

Residual analysis did not show any violation of the normality assumption, and the residuals show a symmetrical distribution around zero, with an approximately constant variance. This is a necessary step in checking the model's adequacy to the observed data. A final assessment of the model adequacy can be better visualized in Figure 1, by comparing observed and predicted values for both models (fixed and random). Observed data for infants numbered 5 and 9 overlap the population average predicted by the model. This pattern is not observed for other infants.

Both fixed and random effects were statistically significant in the final model (Table 1). Sex was significant in all three parameters: girls' body weights are significantly lower than boys' at birth (-0.0971), as well as their equilibrium body weight $(-0.3639)$ and their tendency to have smaller rates of body weight gain $(-0.0991)$ during the first months of life. Gestational age only affects birth weight (0.1338), that is, higher gestational age implies greater birth weight. Type of delivery has a significant effect $(0.0766)$ on growth rate: infants born through the cesarean delivery method tend to have higher rates of body weight gain than infants born through normal delivery (Table 1 ).

The significant effect of breastfeeding duration on equilibrium body weight $(-0.2813)$ and growth rate $(0.0798)$ of 


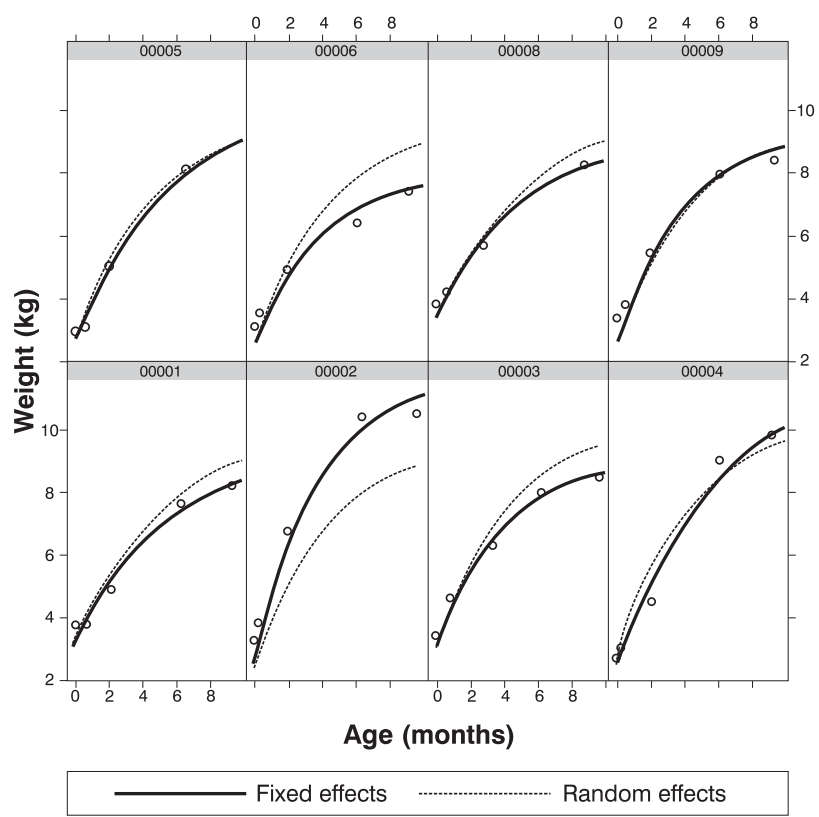

The information on the first eight children with complete follow-up data is shown.

Figure 1 - Adjusted models with fixed and random effects compared with the observed values of infant body weight (Rio de Janeiro, 1999-2001)

infants deserves special attention. Note that although growth rate is higher as predominant breastfeeding duration increases, equilibrium body weight is lower. This means that infants with longer predominant breastfeeding duration achieve a lower equilibrium weight at the end of the study period, although they are subject to a larger growth rate in the first few months of life (Table 1).

In Figure 2, the crossing point corresponds to the ages of 6.75 and 7 months, for boys and girls, respectively. From this time, infants predominantly breastfed for 6 months or more start to have a lower rate of body weight gain than infants breastfed for less than 6 months.

The growth rate is $16.4 \%$ per month, for boys, and $14.8 \%$, for girls. This means that, on average, boys reached half of their equilibrium weight gain in 4.24 months, which is approximately 127 days, compared to 4.68 months (140 days) for girls.

Girls have a significantly lower length at birth (-0.6630) and smaller growth rate $(-0.1218)$ when compared to boys. However, no significant difference for the equilibrium length was observed. Gestational age influences only length at birth (0.6088); the longer the gestational age, the greater length at birth (Table 2).

The breast-feeding duration has a greater effect on the rate of infant growth $(0.0746)$ than on the equilibrium length $(-1.5422)$. Similarly to what was observed for the response variable body weight, the equilibrium length for infants breastfed for a longer period of time is smaller, although this group experienced a higher growth rate in the first months of life (Table 2).

\section{Discussion}

This study shows the important effect of predominant breastfeeding duration on the growth rate of infants as well as on their equilibrium body weight and length. In it, it was observed that although the growth rate is higher in infants with longer predominant breastfeeding duration, their body weight at the end of the study is lower and their body length is smaller than their formula-fed counterparts. It was verified that this latter group grew heavier and bigger than the former, starting around 7 months of life. This occurrence in the third quarter of infants' growth period has been previously discussed in the literature. ${ }^{1,6,7}$ The present study corroborates this hypothesis using a new class of models and it is in accordance with the results reported by the new WHO growth standards recently released ${ }^{8,9}$ and with some other investigations comparing previous growth standards with the current WHO growth charts. ${ }^{19,20}$

Some authors have argued that the difference in growth rate observed between breastfed and formula-fed infants is due either to the excessive feeding of formula-fed infants ${ }^{21}$ or to the high caloric intake of formulas. ${ }^{22-24}$ Dewey et al. ${ }^{1,25}$ observed that formula-fed infants consume more milk and gain body weight more rapidly than breastfed infants, behavior that is prone to a higher obesity risk. These authors stated that blood insulin levels can be affected by feeding practices through protein intake. ${ }^{21}$ Other authors ${ }^{26-28}$ have explained the same process as occurring in reverse causality, that is, deficits on infant growth lead to changes in feeding patterns, favoring complementation or earlier cessation of breastfeeding.

Other important variables were also analyzed. Gestational age affects body weight and length at birth, and does not show a significant effect on the equilibrium body weight and length on the growth rate. With regard to sex, boys tend to weigh more than girls, and to have larger birth weight and a higher rate of body weight gain. Infants who were born through cesarean delivery presented higher weight gain than those born via natural delivery. Although several studies ${ }^{29,30}$ have discussed the relationship between delivery type and breastfeeding duration, none of them has dealt with the influence of delivery type on the evolution of infant growth in the first few months of life.

Previous works published in the literature have addressed the effect of predominant breastfeeding duration on infant growth. However, the statistical procedures used are based on linear models or in logistic regression. These models, although useful and easy to interpret, do not consider the behavior of the underlying mechanism producing the 
Table 1 - Parameters of nonlinear mixed effect model for body weight $(\mathrm{kg})$ of children aged less than 1 year old (Rio de Janeiro, 1999-2001)

\begin{tabular}{|c|c|c|c|}
\hline Fixed effects & Estimates & Standard error & $\mathbf{p}$ \\
\hline$P_{\text {asymp. }}($ intercept) & 11.2074 & 0.1858 & $<0.0001$ \\
\hline$P_{\text {asymp. }}$ sex & -0.3639 & 0.1887 & 0.0541 \\
\hline$P_{\text {asymp. }}$ AMPRED $_{\mathrm{i}}(\mathrm{mo})$ & -0.2813 & 0.0390 & $<0.0001$ \\
\hline$P_{0}$ (intercept) & 3.0985 & 0.0362 & $<0.0001$ \\
\hline$P_{0}$ sex & -0.0971 & 0.0254 & 0.0431 \\
\hline$P_{0}$ gestational age & 0.1338 & 0.0133 & $<0.0001$ \\
\hline Log_rate (intercept) & -1.8104 & 0.0385 & $<0.0001$ \\
\hline Log_rate sex & -0.0991 & 0.0357 & 0.0056 \\
\hline Log_rate AMPRED $_{\mathrm{i}}(\mathrm{mo})$ & 0.0798 & 0.0110 & $<0.0001$ \\
\hline Log_rate delivery type & 0.0766 & 0.0289 & 0.0083 \\
\hline-2 log-likelihood & 2069.3 & & \\
\hline AIC & 2103.3 & & \\
\hline Random effects & & Estimate $(95 \% \mathrm{CI})$ & \\
\hline$\sigma_{\text {asymp. (intercept) }}$ & & $1.2512(1.4170$ to 1.6048$)$ & \\
\hline$\sigma_{\mathrm{PO} \text { (intercept) }}$ & & 0.3391 ( 0.3766 to 0.4183$)$ & \\
\hline$\sigma_{\text {log_rate (intercept) }}$ & & $0.1312(0.1722$ to 0.2260$)$ & \\
\hline Cor $\left[\right.$ Asymp., $\left.P_{0}\right]$ & & $-0.1148(0.0520$ to 0.2159$)$ & \\
\hline Cor [Asymp., log_rate] & & $-0.5666(-0.3882$ to -0.1750$)$ & \\
\hline $\operatorname{Cor}\left[P_{0}\right.$, log_rate $]$ & & $0.0393(0.3376$ to 0.5806$)$ & \\
\hline$\sigma_{\text {residual }}$ & & $0.2514(0.2669$ to 0.2834$)$ & \\
\hline
\end{tabular}

$95 \% \mathrm{CI}=95 \%$ confidence interval; $\mathrm{AIC}=$ Akaike information criterion; $\mathrm{AMPRED}_{\mathrm{i}}=$ predominant breast-feeding duration; fixed effect $=$ reflect the overall average profile; log_rate = logarithm of the rate of body weight gain or length gain; $P_{\text {asymp. }}=$ equilibrium body weight or length at the end of the study period; $P_{0}=$ body weight or length at birth; random effect $=$ reflect how subject-specific profiles deviate from the overall average profile; residual $=$ unexplained variation.

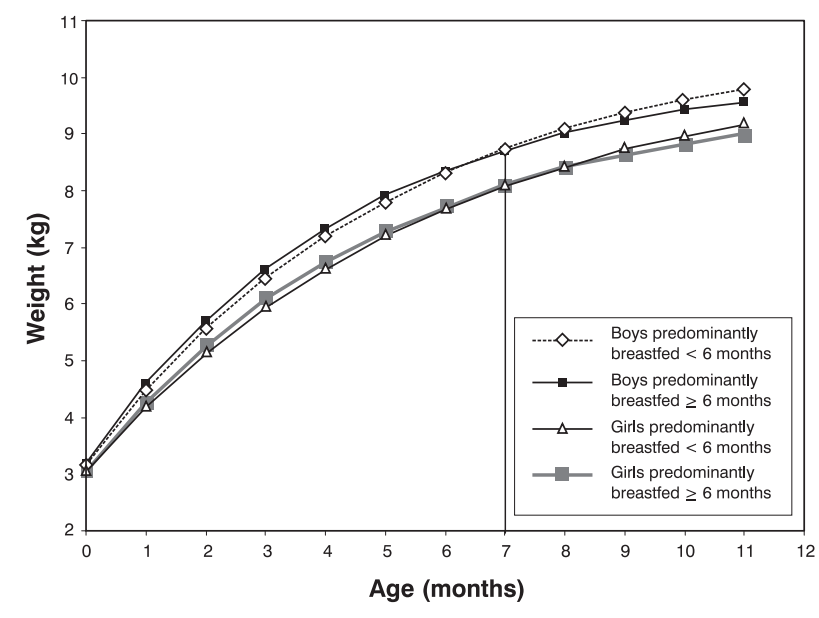

Figure 2 - Body weight $(\mathrm{kg})$ as a function of predominantly breastfed groups stratified by sex (Rio de Janeiro, 1999-2001)

response variable, which in this case refers to the body weight and length of infants in the first months of life. In the great majority of studies regarding growth models, the interest lies on fitting individual curves. In this context, random effect models are especially attractive given their model components. This procedure increases the precision of estimated parameters.

Some limitations of the present study must be addressed. Although prospective data analysis is sensitive to follow-up losses, fortunately this was not the case in the present study. Statistical analyses indicated that follow-up losses were random according to several variables of interest, such as age category, skin color, schooling and others. ${ }^{15}$ Another possible limitation originates from the use of weight and length at birth as reported by the mother. However, they were captured in the first interview and did not show inconsistencies with the other observed measures, which is an indication of the quality of reported data.

In conclusion, the present study confirms the differences observed in infant growth based on different feeding practices between the sixth and the seventh month of life and it is consistent with the results observed by WHO new growth standards. We believe that the merit of the current paper is its potential contribution to the dissemination of a new 
Table 2 - Parameter estimates for the nonlinear mixed effects model for length (cm) for infants aged less than 1 year old (Rio de Janeiro, 1999-2001)

\begin{tabular}{|c|c|c|c|}
\hline Fixed effects & Estimate & Standard error & $\mathbf{p}$ \\
\hline Asymp. (intercept) & 86.0871 & 1.0226 & $<0.0001$ \\
\hline Asymp. AMPRED ${ }_{i}(m o)$ & -1.5422 & 0.2208 & $<0.0001$ \\
\hline$P_{0}$ (intercept) & 49.1013 & 0.2961 & $<0.0001$ \\
\hline$P_{0}$ sex & -0.6630 & 0.1847 & 0.0431 \\
\hline$P_{0}$ gestational age & 0.6088 & 0.0481 & $<0.0001$ \\
\hline log_rate (intercept) & -2.1667 & 0.0514 & $<0.0001$ \\
\hline log_rate sex & -0.1218 & 0.0173 & 0.0056 \\
\hline $\log _{\text {rate }} \mathrm{AMPRED}$ ( $(\mathrm{mo})$ & 0.0746 & 0.0119 & $<0.0001$ \\
\hline-2 log-likelihood & 6619.2 & & \\
\hline AIC & 6649.2 & & \\
\hline Random effects & & Estimate $(95 \% \mathrm{CI})$ & \\
\hline$\sigma_{\text {asymp. (intercept) }}$ & & 0.9952 (1.6151 to 2.6210$)$ & \\
\hline$\sigma_{\mathrm{PO} \text { (intercept) }}$ & & $1.5260(1.6654$ to 1.8176$)$ & \\
\hline$\sigma_{\text {log_rate (intercept) }}$ & & 0.0832 (0.1111 to 0.1483$)$ & \\
\hline $\operatorname{Cor}\left[\right.$ Asymp., $\left.P_{0}\right]$ & & $0.1334(0.4566$ to 0.6920$)$ & \\
\hline Cor [Asymp., log_rate] & & $-0.1008(0.0038$ to 0.1085$)$ & \\
\hline $\operatorname{Cor}\left[P_{0}\right.$, log_rate $]$ & & $-0.1033(0.0053$ to 0.1138$)$ & \\
\hline$\sigma_{\text {residual }}$ & & $1.1116(1.1691$ to 1.2186$)$ & \\
\hline
\end{tabular}

$95 \% \mathrm{CI}=95 \%$ confidence interval $;$ AIC $=$ Akaike Information Criterion $;$ AMPRED $_{i}=$ predominant breast-feeding duration; fixed effect $=$ reflect the overall average profile; log_rate $=$ logarithm of the rate of body weight or length gain; $P_{\text {asymp. }}=$ equilibrium body weight or length at the end of the study period; $P_{0}=$ body weight or length at birth; random effect = reflect how subject-specific profiles deviate from the overall average profile; residual = unexplained variation.

approach which might facilitate the analysis and interpretation of growth data for both individuals and populations.

\section{Acknowledgements}

MHCS participated in the statistical analysis and in the writing of the manuscript. GK participated in all phases of the study, including its conception, drawing, follow-up, data interpretation and writing of the manuscript. CJS participated in several phases of the study, including data interpretation and writing of the manuscript. MTSB participated in data interpretation and writing of the manuscript. There are no conflicts of interest by the authors of this work.

\section{References}

1. Dewey KG. Growth characteristics of breast-fed compared to formula-fed infants. Biol Neonate. 1998;74:94-105.

2. Hop LT, Gross R, Giay T, Sastroamidjojo S, Schultink W, Lang NT. Premature complementary feeding is associated with poorer growth of Vietnamese children. J Nutr. 2000;130: 2683-90.

3. Fewtrell MS, Lucas A, Morgan JB. Factors associated with weaning in full term and preterm infants. Arch Dis Child Fetal Neonatal Ed. 2003;88:F296-301.
4. Kramer MS, Kakuma R. Optimal duration of exclusive breastfeeding. Cochrane Database Syst Rev. 2002:CD003517.

5. Kramer MS, Guo T, Platt RW, Sevkovskaya Z, Dzikovich I, Collet JP, et al. Infant growth and health outcomes associated with 3 compared with 6 mo of exclusive breastfeeding. Am J Clin Nutr. 2003;78:291-5.

6. de Onis M, Onyango AW. The Centers for Disease Control and Prevention 2000 growth charts and the growth of breastfed infants. Acta Paediatr. 2002;92:413-9.

7. Donma MM, Donma O. Infant feeding and growth: a study on Turkish infants from birth to 6 months. Pediatr Int. 1999;41: 542-8.

8. WHO Multicentre Growth Reference Study Group. WHO Child Growth Standards: length/height-for-age, weight-for-age, weight-for length, weight-for-height and body mass index-for-age: methods and development. Geneva: World Health Organization; 2006.

9. WHO Multicentre Growth Reference Study Group. WHO child growth standards based on length/height, weight and age. Acta Paediatr Suppl. 2006;450:76-85.

10. Pinheiro JC, Bates DM. Mixed-effects models in S and S-PLUS. New York: Springer; 2000.

11. Kac G. Fatores determinantes de retenção de peso pós-parto em uma coorte de mulheres com nove meses de seguimento [tese]. São Paulo: Universidade de São Paulo; 2002. 
12. Kac G, Benício MH, Valente JG, Velásquez-Meléndez G. Postpartum weight retention among women in Rio de Janeiro: a follow-up study. Cad Saude Publica. 2003;19:S149-61.

13. Kac G, Benício MH, Velásquez-Meléndez G, Valente JG, Struchiner $\mathrm{CJ}$. Breastfeeding and postpartum weight retention in a cohort of Brazilian women. Am J Clin Nutr. 2004;79:487-93.

14. Kac G, Benício MH, Velásquez-Meléndez G, Valente JG, Struchiner $\mathrm{CJ}$. Gestational weight gain and prepregnancy weight influence postpartum weight retention in a cohort of Brazilian women. J Nutr. 2004;134:661-6.

15. Spyrides MH, Struchiner CJ, Barbosa MT, Kac G. Práticas de amamentação e crescimento infantil: um estudo longitudinal em crianças do Rio de Janeiro, 1999/ 2001. Cad Saude Publica. 2005; 21:756-66.

16. World Health Organization (WHO). Physical status: the use and interpretation of anthropometry. WHO Technical Report Series 854. Geneva: World Health Organization; 1995.

17. Lohman TG, Roche AF, Martorell R. Anthropometric standardization reference manual. Champaign, IL: Human Kinetics Books; 1988.

18. OPAS/OMS. Indicadores para evaluar las prácticas de lactancia materna. Informe de una reunión de 11-12 de junio. Ginebra, Suiza: OMS; 1991.

19. de Onis M, Onyango AW, Borghi E, Garza C, Yang H; WHO Multicentre Growth Reference Study Group. Comparison of the WHO child growth standards and the CDC 2000 growth charts. J Nutr. 2007;137:144-8.

20. de Onis M, Onyango AW, Borghi E, Garza C, Yang H; WHO Multicentre Growth Reference Study Group. Comparison of the World Health Organization (WHO) child growth standards and the National Center for Health Statistics/WHO international growth reference: implications for child health programmes. Public Health Nutr. 2006;9:942-7.

21. Yoneyama K, Nagata H, Asano H. Growth of Japanese breast-fed and bottle-fed infants from birth to 20 months. Ann Hum Biol. 1994;21:597-608.
22. Axelsson I, Borulf S, Righard L, Raiha N. Protein and energy intake during weaning: I. Effects on growth. Acta Paediatr Scand. 1987;76:321-7.

23. Butte NF, Wong WW, Garza C, Stuff JE, Smith EO, Klein PD, et al. Energy requirements of breast-fed infants. J Am Coll Nutr. 1991;10:190-5.

24. Butte NF, Wong WW, Hopkinson JM, Heinz CJ, Mehta NR, Smith EO. Energy requirements derived from total energy expenditure and energy deposition during the first 2 y of life. Am J Clin Nutr. 2000;72:1558-69.

25. Dewey KG. Is breastfeeding protective against child obesity? J Hum Lact. 2003;19:9-18.

26. Galler JR, Ramsey FC, Harrison RH, Brooks R, Weiskopf-Bock S. Infant feeding practices in Barbados predict later growth. J Nutr. 1998;128:1328-35.

27. Marquis GS, Habicht JP, Lanata CF, Black RE, Rasmussen KM. Association of breastfeeding and stunting in Peruvian toddlers: an example of reverse causality. Int J Epidemiol. 1997;26: 349-56.

28. Victora CG, Morris SS, Barros FC, Horta BL, Weiderpass E, Tomasi E. Breast-feeding and growth in Brazilian infants. Am J Clin Nutr. 1998;67:452-8.

29. Perez-Escamilla R, Maulen-Radovan I, Dewey KG. The association between cesarean delivery and breast-feeding outcomes among Mexican women. Am J Pub Health. 1996;86:832-6.

30. Chapman DJ, Perez-Escamilla R. Does delayed perception of the onset of lactation shorten breastfeeding duration? J Hum Lact. 1999;15:107-11.

Correspondence:

Gilberto Kac

Universidade Federal do Rio de Janeiro

Instituto de Nutrição Josué de Castro

Departamento de Nutrição Social e Aplicada

Av. Brigadeiro Trompowsky s/n ${ }^{\circ}$, bloco $\mathrm{J}, 2^{\circ}$ andar

CEP 21941-590 - Rio de Janeiro, RJ - Brazil

E-mail: kacetal@gmail.com,gkac@nutricao.ufrj.br 